\title{
Cohomological rigidity of real Bott manifolds
}

\author{
YOSHINOBU KAMISHIMA \\ MIKIYA MASUDA
}

\begin{abstract}
A real Bott manifold is the total space of an iterated $\mathbb{R} \mathbb{P}^{1}$-bundle over a point, where each $\mathbb{R} \mathbb{P}^{1}$-bundle is the projectivization of a Whitney sum of two real line bundles. We prove that two real Bott manifolds are diffeomorphic if their cohomology rings with $\mathbb{Z} / 2$-coefficients are isomorphic.

A real Bott manifold is a real toric manifold and admits a flat Riemannian metric invariant under the natural action of an elementary abelian 2-group. We also prove that the converse is true, namely a real toric manifold which admits a flat Riemannian metric invariant under the action of an elementary abelian 2-group is a real Bott manifold.
\end{abstract}

57R91; 53C25, 14M25

\section{Introduction}

A fundamental result in the theory of toric varieties says that the categories of toric varieties (over the complex numbers $\mathbb{C}$ ) and fans are equivalent (see Oda [20]). This reduces the classification of toric varieties to that of fans. Among toric varieties, compact smooth toric varieties which we call toric manifolds are well studied and the classification as varieties is completed for some classes of toric manifolds (see Kleinschmidt [11], Oda [20], Sato [22] for example).

However, not much is known for the topological classification of toric manifolds, and the following problem is addressed by Masuda and Suh in [17] (see also Choi, Masuda and Suh [6], Masuda and Panov [16]).

Cohomological rigidity problem for toric manifolds Are two toric manifolds diffeomorphic (or homeomorphic) if their cohomology rings with integer coefficients are isomorphic as graded rings?

As is well-known, there are many closed smooth manifolds which are not homeomorphic but have isomorphic cohomology rings. So the problem above seems unlikely to have a 
positive answer, but no counterexample is known and there are some partial affirmative solutions to the problem (see $[6 ; 16 ; 17])$.

The set $X(\mathbb{R})$ of real points in a toric manifold $X$ is called a real toric manifold. It appears as the fixed point set of complex conjugation on $X$. For example, when $X$ is a complex projective space $\mathbb{C P}^{n}$, the subspace $X(\mathbb{R})$ is a real projective space $\mathbb{R P}^{n}$. It is known that

$$
H^{*}(X(\mathbb{R}) ; \mathbb{Z} / 2) \cong H^{2 *}(X ; \mathbb{Z}) \otimes \mathbb{Z} / 2 \text { as graded rings }
$$

for any toric manifold $X$ where $\mathbb{Z}$ denotes the integers and $\mathbb{Z} / 2=\{0,1\}$, and one may ask the same question as the rigidity problem above for real toric manifolds with $\mathbb{Z} / 2$-coefficients, namely:

Cohomological rigidity problem for real toric manifolds Are two real toric manifolds diffeomorphic (or homeomorphic) if their cohomology rings with $\mathbb{Z} / 2$-coefficients are isomorphic as graded rings?

In this paper we are concerned with a sequence of $\mathbb{R P}^{1}$-bundles

$$
M_{n} \stackrel{\mathbb{R P}^{1}}{\longrightarrow} M_{n-1} \stackrel{\mathbb{R P}^{1}}{\longrightarrow} \cdots \stackrel{\mathbb{R P}^{1}}{\longrightarrow} M_{1} \stackrel{\mathbb{R P}^{1}}{\longrightarrow} M_{0}=\{\text { a point }\}
$$

such that $M_{i} \rightarrow M_{i-1}$ for $i=1, \ldots, n$ is the projective bundle of a Whitney sum of two real line bundles over $M_{i-1}$, where one of the two line bundles may be assumed to be trivial without loss of generality because projectivizations $P(E)$ and $P(E \otimes L)$ are diffeomorphic for any real vector bundle $E$ and line bundle $L$ over a smooth manifold. Grossberg and Karshon [9] considered the sequence above in the complex case and named it a Bott tower of height $n$. Following them, we call the sequence above a real Bott tower of height $n$. Each $M_{i}$ in the tower (1-1) is a real toric manifold. We call $M_{i}$ a real Bott manifold. There are many choices of line bundles in the tower (1-1) so that real Bott towers produce many real Bott manifolds. We note that even if real Bott towers of height $n$ are different, their top manifolds of dimension $n$ might be diffeomorphic.

The main purpose of this paper is to prove the following which answers the cohomological rigidity problem affirmatively for real Bott manifolds.

Theorem 1.1 Two real Bott manifolds are diffeomorphic if their cohomology rings with $\mathbb{Z} / 2$-coefficients are isomorphic as graded rings.

Although real toric manifolds have similar properties to toric manifolds, there is one major difference, which is that a real toric manifold is not simply connected while a 
toric manifold is simply connected. The universal cover of a real Bott manifold is a Euclidean space so that a real Bott manifold is aspherical. In fact, we will see that real Bott manifolds are flat Riemannian manifolds.

Any real toric manifold of dimension $n$ supports an action of an elementary abelian 2group $T^{n}(\mathbb{R})$ of rank $n$ and real Bott manifolds of dimension $n$ admit a flat Riemannian metric invariant under the action of $T^{n}(\mathbb{R})$. The following shows that these are the only examples among real toric manifolds.

Theorem 1.2 A real toric manifold of dimension $n$ which admits a flat Riemannian metric invariant under the action of $T^{n}(\mathbb{R})$ is a real Bott manifold.

This paper is organized as follows. We describe the cohomology ring and the fundamental group of a real Bott manifold in Sections 2 and 3. In Section 4 we find necessary and sufficient conditions for an isomorphism between cohomology rings of real Bott manifolds to satisfy in terms of matrices. Using these conditions, we construct in Section 5 a monomorphism between the fundamental groups of the real Bott manifolds. It may not be an isomorphism but the existence of the monomorphism implies that the fundamental groups are isomorphic, which is proved in Section 6 by studying group extensions. Since real Bott manifolds are flat Riemannian manifolds, the isomorphism of the fundamental groups implies Theorem 1.1 by the Bieberbach theorem. In Section 7 we enumerate diffeomorphism classes in real Bott manifolds of dimension up to 4 . This result is obtained by Nazra in [19] independently by a different method. Theorem 1.2 is proved in Section 8. In Section 9 we view real Bott manifolds from the viewpoint of small covers introduced by Davis and Januszkiewic in [7]. In the Appendix, we give a proof of a (probably known) fact used in Section 6.

Throughout the paper we write $\mathbb{Z} / 2$ for the additive group $\{0,1\}$ and $\mathbb{Z}_{2}$ for the multiplicative group $\{ \pm 1\}$.

\section{Cohomology rings}

We shall describe the cohomology ring of the real Bott manifold $M_{n}$ in the tower (1-1). We recall certain well-known facts from Borel and Hirzebruch [1, 15.4]. Let $E \rightarrow X$ be a real vector bundle of rank $m$ over a topological space $X$ and let $P(E)$ be the projectivization of $E$. Then $H^{*}(P(E) ; \mathbb{Z} / 2)$ is an algebra over $H^{*}(X)$ through the projection map from $P(E)$ to $X$ and the algebra structure is described as

$$
H^{*}(P(E) ; \mathbb{Z} / 2)=H^{*}(X ; \mathbb{Z} / 2)[x] /\left(\sum_{i=0}^{m} w_{i}(E) x^{m-i}\right)
$$


where $w_{i}(E)$ denotes the $i^{\text {th }}$ Stiefel-Whitney class of $E$ and $x$ is given by the first Stiefel-Whitney class of the canonical line bundle over $P(E)$. Moreover, the total Stiefel-Whitney class of $T_{f} P(E)$ the tangent bundle along the fibers of $P(E)$ is given by

$$
w\left(T_{f}(P(E))\right)=\sum_{i=0}^{m} w_{i}(E)(1+x)^{m-i}
$$

in particular,

$$
w_{1}\left(T_{f}(P(E))\right)=w_{1}(E)
$$

when $m$ is even.

Now we return to the tower (1-1). By definition $M_{j}=P\left(L_{j-1} \oplus \mathbb{R}\right)$ for some line bundle $L_{j-1}$ over $M_{j-1}$ for $j=1, \ldots, n$, where $\mathbb{R}$ denotes the trivial line bundle. Let $\gamma_{j}$ be the canonical line bundle over $M_{j}$ and set $x_{j}=w_{1}\left(\gamma_{j}\right)$. We use the same notation $\gamma_{j}$ (resp. $x_{j}$ ) for the pullback of $\gamma_{j}$ (resp. $x_{j}$ ) by compositions of projections $M_{k} \rightarrow M_{k-1} \rightarrow \cdots \rightarrow M_{j}$ where $k>j$. Then repeated use of (2-1) shows

$$
H^{*}\left(M_{k} ; \mathbb{Z} / 2\right)=\mathbb{Z} / 2\left[x_{1}, \ldots, x_{k}\right] /\left(x_{j}\left(x_{j}+w_{1}\left(L_{j-1}\right)\right) \mid j=1, \ldots, k\right) .
$$

Since $H^{1}\left(M_{j-1} ; \mathbb{Z} / 2\right)$ is additively generated by $x_{1}, \ldots, x_{j-1}$ and $L_{j-1}$ is a line bundle over $M_{j-1}$, one can uniquely write

$$
w_{1}\left(L_{j-1}\right)=\sum_{i=1}^{j-1} A_{j}^{i} x_{i} \quad \text { with } A_{j}^{i} \in \mathbb{Z} / 2
$$

where $j=2, \ldots, n$. Line bundles are classified by their first Stiefel-Whitney classes and the first Stiefel-Whitney class behaves additively for tensor products of line bundles; so it follows from (2-4) that

$$
L_{j-1}=\gamma_{1}^{A_{j}^{1}} \otimes \cdots \otimes \gamma_{j-1}^{A_{j}^{j-1}}
$$

For convenience, we set $A_{j}^{i}=0$ unless $i<j$ and form a square matrix $A$ of size $n$ with $A_{j}^{i}$ as the $(i, j)$ entry. So $A$ is an upper triangular matrix with zero diagonal entries.

Observation (2-5) implies that the tower (1-1) is completely determined by the matrix $A$. So we may denote $M_{n}$ by $M(A)$. For later use we record the ring structure of $H^{*}(M(A) ; \mathbb{Z} / 2)$ as a lemma which follows from (2-3) and (2-4). 
Lemma 2.1 Let $A$ and $M(A)$ be as above. Then $H^{*}(M(A) ; \mathbb{Z} / 2)$ is generated by degree one elements $x_{1}, \ldots, x_{n}$ as a graded ring with $n$ relations

$$
x_{j}^{2}=x_{j} \sum_{i=1}^{n} A_{j}^{i} x_{i} \quad \text { for } j=1, \ldots, n \text {. }
$$

We conclude this section with the following lemma.

Lemma 2.2 The real Bott manifold $M(A)$ is orientable if and only if the sum of entries is zero in $\mathbb{Z} / 2$ for each row of $A$.

Proof The repeated use of (2-2) together with (2-4) shows that

$$
\begin{aligned}
w_{1}(M(A)) & =\sum_{j=1}^{n} w_{1}\left(L_{j-1} \oplus \underline{\mathbb{R}}\right)=\sum_{j=1}^{n} w_{1}\left(L_{j-1}\right) \\
& =\sum_{j=1}^{n} \sum_{i=1}^{j-1} A_{j}^{i} x_{i}=\sum_{i=1}^{n}\left(\sum_{j=1}^{n} A_{j}^{i}\right) x_{i} .
\end{aligned}
$$

Since $M(A)$ is orientable if and only if $w_{1}(M(A))=0$, the lemma follows from the identity above.

An analogous statement to Lemma 2.2 holds for any real toric manifold and for SUstructures of toric manifolds (see Buchstaber [2, page 23]).

\section{Fundamental groups}

A general description of the fundamental group of an arbitrary real toric manifold is given by Uma in [23] motivated by the work of Davis and Januszkiewic [7]. In this section, we shall describe the fundamental group of $M(A)$ in a direct way.

Let $s_{i}(i=1, \ldots, n)$ be a Euclidean motion on $\mathbb{R}^{n}$ defined by

$$
\begin{aligned}
s_{i}\left(u_{1}, \ldots, u_{n}\right) & =\left(u_{1}, \ldots, u_{i-1}, u_{i}+\frac{1}{2},(-1)^{A_{i+1}^{i}} u_{i+1}, \ldots,(-1)^{A_{n}^{i}} u_{n}\right) \\
& =\left((-1)^{A_{1}^{i}} u_{1}, \ldots,(-1)^{A_{n}^{i}} u_{n}\right)+\frac{1}{2} e^{i}
\end{aligned}
$$

where $e^{1}, \ldots, e^{n}$ denote the standard basis of $\mathbb{R}^{n}$. The group $\Gamma(A)$ generated by $s_{1}, \ldots, s_{n}$ contains all translations by $\mathbb{Z}^{n}$ which are generated by $s_{1}^{2}, \ldots, s_{n}^{2}$. The action of $\Gamma(A)$ on $\mathbb{R}^{n}$ is free and the orbit space $\mathbb{R}^{n} / \Gamma(A)$ is compact. Since the action of $\Gamma(A)$ on $\mathbb{R}^{n}$ preserves the Euclidean metric on $\mathbb{R}^{n}$ which is flat, the orbit space $\mathbb{R}^{n} / \Gamma(A)$ inherits the flat metric. 
Lemma 3.1 $\mathbb{R}^{n} / \Gamma(A)$ is diffeomorphic to $M(A)$. Therefore $M(A)$ is a Riemannian flat manifold with $\Gamma(A)$ as the fundamental group.

Proof Let $\Gamma_{k}(k=1, \ldots, n)$ be a subgroup of $\Gamma(A)$ generated by $s_{1}, \ldots, s_{k}$. It acts on the coordinate subspace $\mathbb{R}^{k}$ of $\mathbb{R}^{n}$ with $u_{k+1}=\cdots=u_{n}=0$ by restricting the action of $\Gamma(A)$ on $\mathbb{R}^{n}$. We claim that a sequence of projections

$$
\mathbb{R}^{n} / \Gamma_{n} \rightarrow \mathbb{R}^{n-1} / \Gamma_{n-1} \rightarrow \cdots \rightarrow \mathbb{R}^{1} / \Gamma_{1} \rightarrow\{0\}
$$

agrees with the real Bott tower (1-1). The lemma follows from the claim.

We shall prove the claim by induction on height. It is obviously true up to height one. Suppose it is true up to height $j-1$. We note that the line bundle $\gamma_{i}$ over $M_{j-1}$ for $i \leq j-1$ is obtained as the quotient of $\mathbb{R}^{j-1} \times \mathbb{R}$ by the diagonal action of $\Gamma_{j-1}$ where the action of $\Gamma_{j-1}$ on the second factor $\mathbb{R}$ is given through a homomorphism $\Gamma_{j-1} \rightarrow \mathbb{Z}_{2}=\{ \pm 1\}$ sending $s_{i}$ to -1 and the others $s_{\ell}(\ell \neq i)$ to 1 . This together with (2-5) shows that the line bundle $L_{j-1}$ in (2-5) is obtained as the quotient of $\mathbb{R}^{j-1} \times \mathbb{R}$ by the diagonal action of $\Gamma_{j-1}$ where the action of $\Gamma_{j-1}$ on the second factor $\mathbb{R}$ is given through a homomorphism $\Gamma_{j-1} \rightarrow \mathbb{Z}_{2}$ sending $s_{i}$ to $(-1)^{A_{j}^{i}}$ for $i \leq j-1$. Therefore the action of $\Gamma_{j-1}$ on $\mathbb{R}^{j-1} \times \mathbb{R}=\mathbb{R}^{j}$ is nothing but the restriction of the action of $\Gamma_{j}$ to $\Gamma_{j-1}$ while the action of $s_{j}$ on $\mathbb{R}^{j}$ is trivial on the first $(j-1)$ coordinates and translation by $1 / 2$ on the last coordinate.

We consider the map

$$
\begin{aligned}
\mathbb{R}^{j}=\mathbb{R}^{j-1} \times \mathbb{R} & \rightarrow\left(\mathbb{R}^{j-1} \times \mathbb{R}\right) / \Gamma_{j-1} \oplus \underline{\mathbb{R}}=L_{j-1} \oplus \underline{\mathbb{R}} ; \\
(x, \theta) & \mapsto([x, \sin 2 \pi \theta], \cos 2 \pi \theta) .
\end{aligned}
$$

Since $s_{i}(x, \theta)=\left(s_{i} x,(-1)^{A_{j}^{i}} \theta\right)$ for $i \leq j-1$ and $s_{j}^{2}(x, \theta)=(x, \theta+1)$, the map above is invariant under the action of $\Gamma_{j-1}$ and $s_{j}^{2}$ and factors through a diffeomorphism from the orbit space $\mathbb{R}^{j} /\left\langle\Gamma_{j-1}, s_{j}^{2}\right\rangle$ onto the unit circle bundle of $L_{j-1} \oplus \underline{\mathbb{R}}$. Furthermore, since $\Gamma_{j}=\left\langle\Gamma_{j-1}, s_{j}\right\rangle$ and $s_{j}(x, \theta)=\left(x, \theta+\frac{1}{2}\right)$, the map induces a diffeomorphism from $\mathbb{R}^{j} / \Gamma_{j}$ onto the projectivization $P\left(L_{j-1} \oplus \underline{\mathbb{R}}\right)=M_{j}$. This shows that the projection $\mathbb{R}^{j} / \Gamma_{j} \rightarrow \mathbb{R}^{j-1} / \Gamma_{j-1}$ agrees with the projection $M_{j} \rightarrow M_{j-1}$, completing the induction step.

We shall investigate the structure of $\Gamma(A)$.

Lemma 3.2 For $i<\ell, s_{\ell} s_{i}=s_{i} s_{\ell}^{(-1)^{A_{\ell}^{i}}}$, ie

$$
s_{\ell} s_{i}= \begin{cases}s_{i} s_{\ell}^{-1} & \text { if } A_{\ell}^{i}=1, \\ s_{i} s_{\ell} & \text { if } A_{\ell}^{i}=0 .\end{cases}
$$


Proof The proof is by direct calculation and left to the reader.

Lemma 3.3 Let $\mathcal{G}(A)$ be the group generated by $\sigma_{1}, \ldots, \sigma_{n}$ and relations given by Lemma 3.2 for $\sigma_{j}$ 's instead of $s_{j}$ 's. Then the homomorphism $\psi: \mathcal{G}(A) \rightarrow \Gamma(A)$ defined by $\psi\left(\sigma_{j}\right)=s_{j}$ for $j=1, \ldots, n$ is an isomorphism.

Proof Using the relations, one can express an element $\sigma$ of $\mathcal{G}(A)$ as $\sigma_{1}^{a_{1}} \sigma_{2}^{a_{2}} \ldots \sigma_{n}^{a_{n}}$ with $a_{1}, \ldots, a_{n} \in \mathbb{Z}$. Suppose $\psi(\sigma)=s_{1}^{a_{1}} s_{2}^{a_{2}} \ldots s_{n}^{a_{n}}$ is the identity element. Then $\psi(\sigma)$ fixes any element of $\mathbb{R}^{n}$. But it maps the origin of $\mathbb{R}^{n}$ to $\frac{1}{2} \sum_{j=1}^{n} \epsilon_{j} a_{j} e_{j}$, where $\epsilon_{j}= \pm 1$, and the image must again be the origin, so we have $a_{j}=0$ for any $j$. This shows that $\sigma$ is the identity and $\psi$ is injective. The surjectivity of $\psi$ is trivial.

The Euclidean motion $s_{j}^{2}$ is a translation of $\mathbb{R}^{n}$ by $e_{j}$ so that $s_{1}^{2}, \ldots, s_{n}^{2}$ commute with each other and generate a free abelian subgroup $N$ of rank $n$. The images of the $s_{j}$ 's in the quotient $\Gamma(A) / N$ commute with each other, which easily follows from Lemma 3.2 , so that $\Gamma(A) / N$ is an elementary abelian 2 -group. We identify $N$ with $\mathbb{Z}^{n}$ and $\Gamma(A) / N$ with $\left(\mathbb{Z}_{2}\right)^{n}$ in a natural way and obtain a short exact sequence:

$$
0 \rightarrow \mathbb{Z}^{n} \rightarrow \Gamma(A) \rightarrow\left(\mathbb{Z}_{2}\right)^{n} \rightarrow 1 .
$$

One may think of $M(A)=\mathbb{R}^{n} / \Gamma(A)$ as the orbit space of the torus $\mathbb{R}^{n} / \mathbb{Z}^{n}$ by the induced action of $\Gamma(A) / \mathbb{Z}^{n}=\left(\mathbb{Z}_{2}\right)^{n}$. We shall explicitly describe the action using complex numbers $\mathbb{C}$. Let $S^{1}$ denote the unit circle of $\mathbb{C}$. We identify $\mathbb{R} / \mathbb{Z}$ with $S^{1}$ (and hence $\mathbb{R}^{n} / \mathbb{Z}^{n}$ with $\left(S^{1}\right)^{n}$ ) through the exponential map sending $u \in \mathbb{R}$ to $\exp (2 \pi \sqrt{-1} u) \in \mathbb{C}$. For $z \in S^{1}$ and $a \in \mathbb{Z} / 2=\{0,1\}$ we define

$$
z(a):= \begin{cases}z & \text { if } a=0, \\ \bar{z} & \text { if } a=1 .\end{cases}
$$

Then the induced action of $s_{i}$ defined in (3-1) on $\left(S^{1}\right)^{n}$ is given by

$$
\left(z_{1}, \ldots, z_{n}\right) \rightarrow\left(z_{1}, \ldots, z_{i-1},-z_{i}, z_{i+1}\left(A_{i+1}^{i}\right), \ldots, z_{n}\left(A_{n}^{i}\right)\right) .
$$

\section{Isomorphisms of cohomology rings}

As is described in Lemma 2.1, $H^{*}(M(A) ; \mathbb{Z} / 2)=R_{1}$ is a graded algebra over $\mathbb{Z} / 2$ generated by degree one elements $x_{1}, \ldots, x_{n}$ with $n$ relations

$$
x_{j}^{2}=x_{j} \sum_{i=1}^{n} A_{j}^{i} x_{i} \quad(j=1, \ldots, n) .
$$


The set $V_{1}$ of degree one elements in $R_{1}$ with vanishing squares forms a vector space over $\mathbb{Z} / 2$ of positive dimension. Set $n_{1}=\operatorname{dim} V_{1}$. Permuting the suffices of $x_{1}, \ldots, x_{n}$, we may assume that the first $n_{1}$ elements $x_{1}, \ldots, x_{n_{1}}$ form a basis of $V_{1}$. We consider the quotient graded ring $R_{2}=R_{1} /\left(V_{1}\right)$ where $\left(V_{1}\right)$ denotes the ideal in $R_{1}$ generated by $V_{1}$. Similarly, the set $V_{2}$ of degree one elements in $R_{2}$ with vanishing squares forms a vector space over $\mathbb{Z} / 2$ of positive dimension. Set $n_{2}=\operatorname{dim} V_{2}$. Permuting the suffices of $x_{n_{1}+1}, \ldots, x_{n}$, we may assume that the image of $x_{n_{1}+1}, \ldots, x_{n_{1}+n_{2}}$ in the quotient ring $R_{2}$ forms a basis of $V_{2}$. Then consider the quotient graded ring $R_{3}=R_{2} /\left(V_{2}\right)$ and repeat the same argument, and so on. This procedure will terminate after a finite number, say $q$, of steps, so that we obtain a sequence of natural numbers $\left(n_{1}, \ldots, n_{q}\right)$.

Definition We call the sequence $\left(n_{1}, \ldots, n_{q}\right)$ the type of $A$ or of $H^{*}(M(A) ; \mathbb{Z} / 2)$. We note that type is invariant under graded ring isomorphism of cohomology rings.

The argument above shows that through a suitable permutation of the suffices of $x_{1}, \ldots, x_{n}$ we may assume that the upper triangular matrix $A$ decomposed into $q \times q$ blocks according to the type $\left(n_{1}, \ldots, n_{q}\right)$ has zero matrices of sizes $n_{1}, \ldots, n_{q}$ as the diagonal $q$ blocks, ie:

$$
A=\left(\begin{array}{cccc}
O_{n_{1}} & & & * \\
& O_{n_{2}} & & \\
& & \ddots & \\
0 & & & O_{n_{q}}
\end{array}\right)
$$

where $O_{m}$ denotes the zero matrix of size $m$ and every column vector in the $(i, i+1)-$ block is non-zero for each $i=1, \ldots, q-1$. We note that permuting the suffices of $x_{1}, \ldots, x_{n}$ corresponds to conjugating the matrix $A$ by a permutation matrix. We call the form (4-2) a normal form.

Let $B$ be an upper triangular matrix of the same normal form as (4-2) and let

$$
\varphi: H^{*}(M(A) ; \mathbb{Z} / 2) \rightarrow H^{*}(M(B) ; \mathbb{Z} / 2)
$$

be an isomorphism as graded rings. We denote by $y_{1}, \ldots, y_{n}$ the generators of $H^{*}(M(B) ; \mathbb{Z} / 2)$. Since $\varphi\left(x_{i}\right)^{2}=\varphi\left(x_{i}^{2}\right)=0$ for $1 \leq i \leq n_{1}, \varphi\left(x_{i}\right)$ is a linear combination of $y_{1}, \ldots, y_{n_{1}}$. In general, one easily sees that $\varphi\left(x_{i}\right)$ for $n_{j-1}+1 \leq i \leq n_{j}$ is a linear combination of $y_{1}, \ldots, y_{n_{j}}$. This means that if we view $P \in \operatorname{GL}(n ; \mathbb{Z} / 2)$ defined by

$$
\left(\varphi\left(x_{1}\right), \ldots, \varphi\left(x_{n}\right)\right)=\left(y_{1}, \ldots, y_{n}\right) P
$$


as a $q \times q$ block matrix of type $\left(n_{1}, \ldots, n_{q}\right)$, then $P$ is an upper triangular block matrix. Since $P$ is non-singular, every diagonal block of $P$ is also non-singular. Therefore, we may assume that the diagonal entries of $P$ are all 1 if necessary by permuting the suffices of the generators $y_{i}$ 's in each block. With this understood, we have

Lemma 4.1 $B=P A$ and

$$
P_{j}^{\ell} B_{\ell}^{i}=P_{j}^{i} B_{j}^{\ell}+P_{j}^{\ell} B_{j}^{i}+P_{j}^{\ell} B_{j}^{\ell} B_{\ell}^{i} \quad \text { for } 1 \leq i<\ell \leq n \text { and } 1 \leq j \leq n .
$$

Proof It follows from (4-3) that

$$
\varphi\left(x_{k}\right)=\sum_{i=1}^{n} P_{k}^{i} y_{i} \quad \text { for } \quad k=1, \ldots, n
$$

We plug this in (4-1) mapped by $\varphi$ to obtain

$$
\begin{aligned}
\left(\sum_{i=1}^{n} P_{j}^{i} y_{i}\right)^{2} & =\left(\sum_{i=1}^{n} P_{j}^{i} y_{i}\right)\left(\sum_{k=1}^{n} \sum_{i=1}^{n} A_{j}^{k} P_{k}^{i} y_{i}\right) \\
& =\left(\sum_{i=1}^{n} P_{j}^{i} y_{i}\right)\left(\sum_{i=1}^{n}(P A)_{j}^{i} y_{i}\right) .
\end{aligned}
$$

Comparing the coefficients of $y_{i} y_{j}$ for $i<j$ in both sides above and noting that $P_{j}^{j}=1$ and $(P A)_{j}^{j}=0$, we obtain

$$
B_{j}^{i}=(P A)_{j}^{i} \quad \text { for } i<j
$$

(Note that the term $y_{i} y_{j}$ may appear in $y_{j}{ }^{2}$ but not in $y_{i}{ }^{2}$ because $B$ is assumed to be upper triangular.) The identity (4-6) holds even for $i \geq j$ because the both sides then vanish. Therefore $B=P A$.

Replacing $P A$ by $B$ and comparing the coefficients of $y_{i} y_{\ell}$ for $i<\ell$ in both sides of (4-5), we obtain the latter identity in the lemma. (Note that $\left(P_{j}^{\ell}\right)^{2}=P_{j}^{\ell}$ since we are working over $\mathbb{Z} / 2$.)

\section{A monomorphism between fundamental groups}

Let $A, B$ and $P$ be as in Section 4. In this section we construct a monomorphism between the fundamental groups $\Gamma(B)$ and $\Gamma(A)$ using $P$.

Any element $s \in \Gamma(A)$ can be expressed uniquely as $s=s_{1}^{a_{1}} s_{2}^{a_{2}} \ldots s_{n}^{a_{n}}$ with integers $a_{i}$ by Lemma 3.2. We denote the exponent $a_{j}$ of $s_{j}$ by $\mathcal{E}_{j}(s)$. 
Lemma 5.1 If $p_{i}, q_{i} \in \mathbb{Z}$ for $i=1, \ldots, n$, then

$$
\begin{aligned}
& \mathcal{E}_{j}\left(\left(s_{1}^{p_{1}} s_{2}^{p_{2}} \ldots s_{n}^{p_{n}}\right)\left(s_{1}^{q_{1}} s_{2}^{q_{2}} \ldots s_{n}^{q_{n}}\right)\right)=(-1)^{\sum_{k=1}^{j-1} q_{k} B_{j}^{k}} p_{j}+q_{j} \\
& \mathcal{E}_{j}\left(\left(s_{1}^{p_{1}} s_{2}^{p_{2}} \ldots s_{n}^{p_{n}}\right)\left(s_{n}^{-q_{n}} \ldots s_{2}^{-q_{2}} s_{1}^{-q_{1}}\right)\right)=(-1)^{\sum_{k=1}^{j-1} q_{k} B_{j}^{k}}\left(p_{j}-q_{j}\right)
\end{aligned}
$$

Proof Using Lemma 3.2, we see

$$
s_{\ell}^{p} s_{k}^{q}=s_{k}^{q} s_{\ell}^{p(-1)^{q B_{\ell}^{k}}} \quad \text { for } \ell>k, \text { and } p, q \in \mathbb{Z}
$$

and repeated use of this identity implies the lemma.

We use the notation $t_{i}$ in $\Gamma(B)$ for the analogues of the $s_{i}$ in $\Gamma(A)$. We regard $P$ as an integer matrix and define

$$
\rho\left(t_{r}\right)=s_{1}^{P_{1}^{r}} s_{2}^{P_{2}^{r}} \ldots s_{n}^{P_{n}^{r}} \quad(r=1, \ldots, n) .
$$

We shall check that $\rho$ preserves the relations in Lemma 3.2 for $\Gamma(B)$ so that $\rho$ induces a homomorphism from $\Gamma(B)$ to $\Gamma(A)$ by Lemma 3.3. It follows from Lemma 5.1 that

$$
\mathcal{E}_{j}\left(\rho\left(t_{\ell} t_{i}\right)\right)=(-1)^{\sum_{k=1}^{j-1} P_{k}^{i} A_{j}^{k}} P_{j}^{\ell}+P_{j}^{i}=(-1)^{B_{j}^{i}} P_{j}^{\ell}+P_{j}^{i} \in \mathbb{Z}
$$

where we used the fact $P A=B$ and $A_{j}^{k}=0$ for $k \geq j$ in the latter identity. Similarly we have

$$
\mathcal{E}_{j}\left(\rho\left(t_{i} t_{\ell}\right)\right)=(-1)^{B_{j}^{\ell}} P_{j}^{i}+P_{j}^{\ell} \in \mathbb{Z}
$$

and

$$
\mathcal{E}_{j}\left(\rho\left(t_{i} t_{\ell}^{-1}\right)\right)=(-1)^{B_{j}^{\ell}}\left(P_{j}^{i}-P_{j}^{\ell}\right) \in \mathbb{Z} .
$$

Now suppose $i<\ell$. When $B_{\ell}^{i}=0$, we have $t_{\ell} t_{i}=t_{i} t_{\ell}$ by Lemma 3.2 for $\Gamma(B)$ and

$$
P_{j}^{\ell} B_{j}^{i}=P_{j}^{i} B_{j}^{\ell} \in \mathbb{Z} / 2
$$

by Lemma 4.1. An elementary case-by-case check (according to the values of $B_{j}^{i}$ and $B_{j}^{\ell}$ ) shows that (5-6) ensures that the right hand sides at (5-3) and (5-4) coincide. When $B_{\ell}^{i}=1$, we have $t_{\ell} t_{i}=t_{i} t_{\ell}^{-1}$ by Lemma 3.2 for $\Gamma(B)$ and

$$
P_{j}^{\ell}=P_{j}^{i} B_{j}^{\ell}+P_{j}^{\ell} B_{j}^{i}+P_{j}^{\ell} B_{j}^{\ell} \in \mathbb{Z} / 2 \text { for } i<\ell
$$

by Lemma 4.1. A similar elementary case-by-case check shows that (5-7) ensures that the right hand sides at (5-3) and (5-5) coincide. In any case the map $\rho$ preserves the relations for $\Gamma(B)$ and $\Gamma(A)$ and hence induces a homomorphism from $\Gamma(B)$ to $\Gamma(A)$. 
Lemma 5.2 The homomorphism $\rho: \Gamma(B) \rightarrow \Gamma(A)$ satisfies

(1) $\rho\left(\mathbb{Z}^{n}\right) \subset \mathbb{Z}^{n}$ and $\mathbb{Z}^{n} / \rho\left(\mathbb{Z}^{n}\right)$ is of order $\operatorname{det} P$ (which is odd),

(2) $\rho$ induces an isomorphism from $\Gamma(B) / \mathbb{Z}^{n}$ onto $\Gamma(A) / \mathbb{Z}^{n}$.

Therefore $\rho$ is injective, and is an isomorphism if and only if $\operatorname{det} P= \pm 1$.

Proof It follows from (5-3) with $\ell=i$ that

$$
\mathcal{E}_{j}\left(\rho\left(t_{i}^{2}\right)\right)= \begin{cases}2 P_{j}^{i} & \text { if } B_{j}^{i}=0, \\ 0 & \text { if } B_{j}^{i}=1 .\end{cases}
$$

Therefore $\rho$ maps the normal subgroup $\mathbb{Z}^{n}$ of $\Gamma(B)$ to that of $\Gamma(A)$, so that $\rho$ maps the short exact sequence (3-2) for $\Gamma(B)$ to that for $\Gamma(A)$. The above fact (5-8) also shows that the map $\rho$ restricted to $\mathbb{Z}^{n}$ agrees with $P$ for $i, j$ with $B_{j}^{i}=0$, in particular, if we view the restricted map as a block matrix as before, then it is an upper triangular block matrix and the diagonal blocks agree with those of $P$. Therefore the determinant of the restricted map is equal to $\operatorname{det} P$. This proves (1).

On the other hand, it follows from the definition (5-2) of $\rho$ that the map induced from $\rho$ on $\Gamma(B) / \mathbb{Z}^{n} \cong(\mathbb{Z} / 2)^{n}$ is nothing but $P$, so it is an isomorphism, proving (2). These imply that $\rho$ is always injective and an isomorphism if and only if $\operatorname{det} P= \pm 1$.

The abelianization of the fundamental group $\Gamma(A)$ of $M(A)$ composed with the natural homomorphism $H_{1}(M(A) ; \mathbb{Z}) \rightarrow H_{1}(M(A) ; \mathbb{Z} / 2)$ agrees with the epimorphism $\Gamma(A) \rightarrow\left(\mathbb{Z}_{2}\right)^{n} \cong(\mathbb{Z} / 2)^{n}$ in (3-2). Therefore, the homomorphism $\rho: \Gamma(B) \rightarrow \Gamma(A)$ always induces an isomorphism $H_{1}(M(B) ; \mathbb{Z} / 2) \rightarrow H_{1}(M(A) ; \mathbb{Z} / 2)$. However, $\rho$ may not induce an isomorphism of the first homology groups with $\mathbb{Z}$-coefficients.

\section{Group extensions}

It is easy to see that a square $(0,1)$-matrix of size $m$ is in $\operatorname{GL}(m ; \mathbb{Z})$ if and only if it is in $\operatorname{GL}(m ; \mathbb{Z} / 2)$ when $m \leq 3$. Therefore, if $n_{i} \leq 3$ for all $i$, where $\left(n_{1}, \ldots, n_{q}\right)$ is the type of $A$ and $B$, then $\operatorname{det} P= \pm 1$ and $\rho$ in Lemma 5.2 is an isomorphism. In general $\rho$ may not be an isomorphism, but we prove the following using the existence of $\rho$.

Lemma 6.1 If $H^{*}(M(A) ; \mathbb{Z} / 2)$ is isomorphic to $H^{*}(M(B) ; \mathbb{Z} / 2)$ as graded rings, then $\Gamma(A)$ is isomorphic to $\Gamma(B)$. 
Remark The reader will find that the proof of this lemma actually shows that any subgroup of $\Gamma(A)$ with odd index is isomorphic to $\Gamma(A)$.

We admit the lemma above for the moment and complete the proof of Theorem 1.1 in the Introduction.

Proof of Theorem 1.1 Real Bott manifolds are compact Riemannian flat manifolds by Lemma 3.1, hence they are diffeomorphic if and only if their fundamental groups are isomorphic by the Bieberbach theorem (see Wolf [24, Theorem 3.3.1 page 105]). Therefore, Theorem 1.1 follows from Lemma 6.1.

The rest of this section is devoted to the proof of Lemma 6.1. Remember the group extension (3-2).

$$
\phi_{A}:\left(\mathbb{Z}_{2}\right)^{n} \rightarrow \operatorname{Aut}\left(\mathbb{Z}^{n}\right) .
$$

We remark that the $\left(\mathbb{Z}_{2}\right)^{n}$-module $\mathbb{Z}^{n}$ via $\phi_{A}$ decomposes into sum of rank one $\left(\mathbb{Z}_{2}\right)^{n}$-modules, which follows from (3-1). There is a 2-cocycle

$$
f_{A}:\left(\mathbb{Z}_{2}\right)^{n} \times\left(\mathbb{Z}_{2}\right)^{n} \rightarrow \mathbb{Z}^{n}
$$

whose cohomology class $\left[f_{A}\right] \in H_{\phi_{A}}^{2}\left(\left(\mathbb{Z}_{2}\right)^{n} ; \mathbb{Z}^{n}\right)$ represents the group extension (3-2), that is, $\Gamma(A)$ is the set $\mathbb{Z}^{n} \times\left(\mathbb{Z}_{2}\right)^{n}$ with group law:

$$
(\ell, \alpha)(m, \beta)=\left(\ell+\phi_{A}(\alpha)(m)+f_{A}(\alpha, \beta), \alpha \beta\right) .
$$

Similarly we have $\phi_{B}$ and $f_{B}$ for the group $\Gamma(B)$.

Lemma 5.2 shows that there is a commutative diagram:

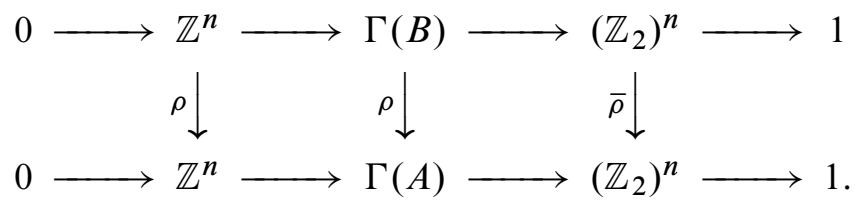

where $\bar{\rho}$ is an isomorphism. We write

$$
\rho(0, \alpha)=(\lambda(\alpha), \bar{\rho}(\alpha)) .
$$

Then, for $(\ell, \alpha) \in \Gamma(B)$ we have

$$
\begin{aligned}
\rho(\ell, \alpha) & =\rho((\ell, 1)(0, \alpha))=\rho(\ell, 1) \rho(0, \alpha) \\
& =(\rho(\ell), 1)(\lambda(\alpha), \bar{\rho}(\alpha)) \\
& =(\rho(\ell)+\lambda(\alpha), \bar{\rho}(\alpha)) .
\end{aligned}
$$


Therefore, applying $\rho$ to the both sides of the equation

$$
(0, \alpha)(0, \beta)=\left(f_{B}(\alpha, \beta), \alpha \beta\right),
$$

we have

$$
\begin{aligned}
\rho((0, \alpha)(0, \beta)) & =(\lambda(\alpha), \bar{\rho}(\alpha))(\lambda(\beta), \bar{\rho}(\beta)) \\
& =\left(\lambda(\alpha)+\phi_{A}(\bar{\rho}(\alpha))(\lambda(\beta))+f_{A}(\bar{\rho}(\alpha), \bar{\rho}(\beta)), \bar{\rho}(\alpha \beta)\right),
\end{aligned}
$$

and

$$
\rho\left(f_{B}(\alpha, \beta), \alpha \beta\right)=\left(\rho\left(f_{B}(\alpha, \beta)\right)+\lambda(\alpha \beta), \bar{\rho}(\alpha \beta)\right)
$$

by (6-3). It follows that

$$
\rho\left(f_{B}(\alpha, \beta)\right)=\lambda(\alpha)+\phi_{A}(\bar{\rho}(\alpha))(\lambda(\beta))-\lambda(\alpha \beta)+\bar{\rho}^{*} f_{A}(\alpha, \beta) .
$$

Similarly, applying $\rho$ to the both sides of the equation

$$
(0, \alpha)(\ell, 1)=\left(\phi_{B}(\alpha)(\ell), \alpha\right),
$$

we have

$$
\begin{aligned}
\rho((0, \alpha)(\ell, 1)) & =(\lambda(\alpha), \bar{\rho}(\alpha))(\rho(\ell), 1) \\
& =\left(\lambda(\alpha)+\phi_{A}(\bar{\rho}(\alpha))(\rho(\ell)), \bar{\rho}(\alpha)\right),
\end{aligned}
$$

and

$$
\rho\left(\phi_{B}(\alpha)(\ell), \alpha\right)=\left(\rho\left(\phi_{B}(\alpha)(\ell)\right)+\lambda(\alpha), \bar{\rho}(\alpha)\right) .
$$

It follows that

$$
\rho\left(\phi_{B}(\alpha)(\ell)\right)=\phi_{A}(\bar{\rho}(\alpha))(\rho(\ell)) .
$$

We regard elements in $\mathbb{Z}^{n}$ as column vectors and represent the homomorphism $\rho: \mathbb{Z}^{n} \rightarrow \mathbb{Z}^{n}$ by an integral matrix $Q$. Then (6-5) is equivalent to

$$
Q \cdot \phi_{B}(\alpha)=\phi_{A}(\bar{\rho}(\alpha)) \cdot Q .
$$

We note that $\widetilde{Q}=(\operatorname{det} Q) Q^{-1}$ is an integral matrix where $\operatorname{det} Q$ is odd, and equal to the order of $\mathbb{Z}^{n} / \rho\left(\mathbb{Z}^{n}\right)$ by Lemma 5.2. It follows from (6-6) that

$$
\phi_{B}(\alpha) \cdot \widetilde{Q}=\widetilde{Q} \cdot \phi_{A}(\bar{\rho}(\alpha)) .
$$

Applying $\widetilde{Q}$ to the both sides of (6-4) and using (6-7), we have

$$
\begin{aligned}
\widetilde{Q} Q f_{B}(\alpha, \beta) & =\widetilde{Q} \lambda(\alpha)+\phi_{B}(\alpha)(\widetilde{Q} \lambda(\beta))-\widetilde{Q} \lambda(\alpha \beta)+\widetilde{Q} \bar{\rho}^{*} f_{A}(\alpha, \beta) \\
& =\delta_{B}(\widetilde{Q} \lambda)(\alpha, \beta)+\widetilde{Q} \bar{\rho}^{*} f_{A}(\alpha, \beta)
\end{aligned}
$$


where we used the definition of the coboundary for the latter identity. Since $\widetilde{Q} Q$ is det $Q$ times the identity matrix, (6-8) implies that

$$
\left[\operatorname{det} Q \cdot f_{B}\right]=\left[\widetilde{Q} \bar{\rho}^{*} f_{A}\right] \in H_{\phi_{B}}^{2}\left(\left(\mathbb{Z}_{2}\right)^{n}, \widetilde{Q} \mathbb{Z}^{n}\right) .
$$

Here $\widetilde{Q} \mathbb{Z}^{n}$ is viewed as a $\left(\mathbb{Z}_{2}\right)^{n}$-module via $\phi_{B}$. It decomposes into the direct sum of rank one $\left(\mathbb{Z}_{2}\right)^{n}$-modules because we have (6-7) and the $\left(\mathbb{Z}_{2}\right)^{n}$-module $\mathbb{Z}^{n}$ via $\phi_{A}$ decomposes into the direct sum of rank one $\left(\mathbb{Z}_{2}\right)^{n}$-modules. Therefore

$$
H_{\phi_{B}}^{2}\left(\left(\mathbb{Z}_{2}\right)^{n}, \widetilde{Q} \mathbb{Z}^{n}\right) \cong \bigoplus_{i=1}^{n} H_{\phi_{i}}^{2}\left(\left(\mathbb{Z}_{2}\right)^{n} ; \mathbb{Z}\right)
$$

where $\phi_{i}:\left(\mathbb{Z}_{2}\right)^{n} \rightarrow \operatorname{Aut}(\mathbb{Z})=\{ \pm 1\}$ is a homomorphism.

Fact $H_{\phi}^{2}\left(\left(\mathbb{Z}_{2}\right)^{n} ; \mathbb{Z}\right)$ is an elementary abelian 2-group for any homomorphism $\phi:\left(\mathbb{Z}_{2}\right)^{n} \rightarrow \operatorname{Aut}(\mathbb{Z})$.

(This fact is probably known but since we do not know the literature, we will give a proof in the Appendix.) It follows from (6-10) and the fact above that $H_{\phi_{B}}^{2}\left(\left(\mathbb{Z}_{2}\right)^{n}, \widetilde{Q} \mathbb{Z}^{n}\right)$ is an elementary abelian 2-group. Since $\operatorname{det} Q$ is odd, (6-9) implies that

$$
\left[f_{B}\right]=\left[\widetilde{Q} \bar{\rho}^{*} f_{A}\right] \in H_{\phi_{B}}^{2}\left(\left(\mathbb{Z}_{2}\right)^{n}, \widetilde{Q} \mathbb{Z}^{n}\right) .
$$

Then $\Gamma(B)$ is isomorphic to the group $\Gamma$ defined by the cocycle $\widetilde{Q} \bar{\rho}^{*} f_{A}$. Using (6-7), $\Gamma$ is the set $\widetilde{Q} \mathbb{Z}^{n} \times\left(\mathbb{Z}_{2}\right)^{n}$ with group law:

$$
\begin{aligned}
(\tilde{Q} \ell, \alpha)(\widetilde{Q} m, \beta) & =\left(\widetilde{Q} \ell+\phi_{B}(\alpha)(\widetilde{Q} m)+\widetilde{Q} f_{A}(\bar{\rho}(\alpha), \bar{\rho}(\beta)), \alpha \beta\right) \\
& =\left(\widetilde{Q}\left(\ell+\phi_{A}(\bar{\rho}(\alpha))(m)+f_{A}(\bar{\rho}(\alpha), \bar{\rho}(\beta))\right), \alpha \beta\right)
\end{aligned}
$$

It suffices to prove that $\Gamma(A)$ is isomorphic to $\Gamma$. Let $\bar{\rho}:\left(\mathbb{Z}_{2}\right)^{n} \rightarrow\left(\mathbb{Z}_{2}\right)^{n}$ be the isomorphism from (6-2). Define a map $\mathcal{T}: \Gamma(A) \rightarrow \Gamma$ by

$$
\mathcal{T}(\ell, \alpha)=\left(\tilde{Q} \ell, \bar{\rho}^{-1}(\alpha)\right) .
$$

This is clearly a bijection. Using (6-11) and (6-1), we have

$$
\begin{aligned}
\mathcal{T}(\ell, \alpha) \mathcal{T}(m, \beta) & =\left(\tilde{Q} \ell, \bar{\rho}^{1}(\alpha)\right)\left(\widetilde{Q} m, \bar{\rho}^{1}(\beta)\right) \\
& =\left(\tilde{Q}\left(\ell+\phi_{A}(\alpha)(m)+f_{A}(\alpha, \beta)\right), \bar{\rho}^{1}(\alpha \beta)\right) \\
& =\mathcal{T}\left(\ell+\phi_{A}(\alpha)(m)+f_{A}(\alpha, \beta), \alpha \beta\right) \\
& =\mathcal{T}((\ell, \alpha)(m, \beta)) .
\end{aligned}
$$

Hence $\mathcal{T}$ is an isomorphism of $\Gamma(A)$ onto $\Gamma$. This completes the proof of Lemma 6.1. 


\section{Classification of real Bott manifolds of low dimension}

Real Bott manifolds are determined by upper triangular square $(0,1)$-matrices with zero diagonal entries, and the diffeomorphism classification of real Bott manifolds reduces to the isomorphism classification of associated cohomology rings with $\mathbb{Z} / 2$-coefficients by our main Theorem 1.1. As observed in Section 4, we may assume that our matrices are of the normal form (4-2). Therefore, it suffices to check which matrices of normal form produce isomorphic cohomology rings and this can be done by case-by-case check when the size $n$ of matrices, that is the dimension of real Bott manifolds, is no greater than 4 . We remember that permuting the suffices of the cohomology generators $x_{1}, \ldots, x_{n}$ in Section 4 corresponds to conjugating our matrices by a permutation matrix. So the cohomology rings associated with conjugate matrices by permutation matrices are isomorphic. This decreases the necessary computations. Below are the results. The same results are obtained by Nazra in [19] independently by studying Seifert fiber structures of real Bott manifolds.

The case $n=2$ Real Bott manifolds of dimension 2 are the torus $\left(S^{1}\right)^{2}$ or the Klein bottle and the corresponding matrices of normal form are respectively the zero matrix of size 2 and $\left(\begin{array}{ll}0 & 1 \\ 0 & 0\end{array}\right)$.

The case $n=3$ There are four diffeomorphism classes of real Bott manifolds of dimension 3, and the corresponding matrices of normal form are distinguished by their types as seen below. Numbers with the superscript $\star$ indicate that the corresponding real Bott manifold is orientable (see Lemma 2.2).

$\mathbf{1}^{\star} \quad$ Type (3)

The zero matrix of size 3. The real Bott manifold is $\left(S^{1}\right)^{3}$.

2 Type $(2,1)$

$$
\left(\begin{array}{lll}
0 & 0 & 0 \\
0 & 0 & 1 \\
0 & 0 & 0
\end{array}\right), \quad\left(\begin{array}{lll}
0 & 0 & 1 \\
0 & 0 & 0 \\
0 & 0 & 0
\end{array}\right), \quad\left(\begin{array}{lll}
0 & 0 & 1 \\
0 & 0 & 1 \\
0 & 0 & 0
\end{array}\right)
$$

where the first and second matrices are conjugate. The real Bott manifold is $S^{1} \times($ Klein bottle).

$\mathbf{3}^{\star}$ Type $(1,2)$

$$
\left(\begin{array}{lll}
0 & 1 & 1 \\
0 & 0 & 0 \\
0 & 0 & 0
\end{array}\right)
$$

4 Type $(1,1,1)$

$$
\left(\begin{array}{lll}
0 & 1 & 0 \\
0 & 0 & 1 \\
0 & 0 & 0
\end{array}\right), \quad\left(\begin{array}{lll}
0 & 1 & 1 \\
0 & 0 & 1 \\
0 & 0 & 0
\end{array}\right)
$$


Remark Compact Riemannian flat manifolds of dimension 3 are classified. There are ten diffeomorphism classes and six of them are orientable (Wolf [24, pages 117 and 120]). One can easily check that the real Bott manifolds in $1^{\star}$ and $3^{\star}$ above are respectively of types $\mathcal{G}_{1}$ and $\mathcal{G}_{2}$ in [24, Theorem 3.5.5] and those in 2 and 4 above are respectively of types $\mathcal{B}_{1}$ and $\mathcal{B}_{3}$ in [24, Theorem 3.5.9].

The case $n=4$ There are twelve diffeomorphism classes of real Bott manifolds of dimension 4, and the corresponding matrices of normal form are as described below. We list representatives of conjugacy classes in matrices of normal form by permutation matrices. The suffix of a matrix below denotes the number of elements in the conjugacy class represented by the matrix. Numbers with the superscript $\star$ indicate orientability, as above.

\section{$\mathbf{1}^{\star} \quad$ Type (4)}

The zero matrix of size 4 . The real Bott manifold is $\left(S^{1}\right)^{4}$.

2 Type $(3,1)$

$$
\left(\begin{array}{llll}
0 & 0 & 0 & 0 \\
0 & 0 & 0 & 0 \\
0 & 0 & 0 & 1 \\
0 & 0 & 0 & 0
\end{array}\right)_{3}\left(\begin{array}{llll}
0 & 0 & 0 & 0 \\
0 & 0 & 0 & 1 \\
0 & 0 & 0 & 1 \\
0 & 0 & 0 & 0
\end{array}\right)_{3}\left(\begin{array}{llll}
0 & 0 & 0 & 1 \\
0 & 0 & 0 & 1 \\
0 & 0 & 0 & 1 \\
0 & 0 & 0 & 0
\end{array}\right)_{1}
$$

The real Bott manifold is $\left(S^{1}\right)^{2} \times($ Klein bottle) .

$\mathbf{3}^{\star}$ Type $(2,2)$

$$
\left(\begin{array}{llll}
0 & 0 & 0 & 0 \\
0 & 0 & 1 & 1 \\
0 & 0 & 0 & 0 \\
0 & 0 & 0 & 0
\end{array}\right)_{2}\left(\begin{array}{llll}
0 & 0 & 1 & 1 \\
0 & 0 & 1 & 1 \\
0 & 0 & 0 & 0 \\
0 & 0 & 0 & 0
\end{array}\right)_{1}
$$

The real Bott manifold is the product of $S^{1}$ and the 3-dimensional real Bott manifold of Type $(1,2)$.

4 Type $(2,2)$

$$
\left(\begin{array}{llll}
0 & 0 & 0 & 1 \\
0 & 0 & 1 & 0 \\
0 & 0 & 0 & 0 \\
0 & 0 & 0 & 0
\end{array}\right)_{2}\left(\begin{array}{llll}
0 & 0 & 1 & 0 \\
0 & 0 & 1 & 1 \\
0 & 0 & 0 & 0 \\
0 & 0 & 0 & 0
\end{array}\right)_{4}
$$

The real Bott manifold is (Klein bottle $) \times($ Klein bottle $)$.

5 Type $(2,1,1)$

$$
\left(\begin{array}{llll}
0 & 0 & 0 & 0 \\
0 & 0 & 1 & 0 \\
0 & 0 & 0 & 1 \\
0 & 0 & 0 & 0
\end{array}\right)_{2}\left(\begin{array}{llll}
0 & 0 & 0 & 0 \\
0 & 0 & 1 & 1 \\
0 & 0 & 0 & 1 \\
0 & 0 & 0 & 0
\end{array}\right)_{2}\left(\begin{array}{llll}
0 & 0 & 1 & 0 \\
0 & 0 & 1 & 0 \\
0 & 0 & 0 & 1 \\
0 & 0 & 0 & 0
\end{array}\right)_{1}\left(\begin{array}{llll}
0 & 0 & 1 & 1 \\
0 & 0 & 1 & 1 \\
0 & 0 & 0 & 1 \\
0 & 0 & 0 & 0
\end{array}\right)_{1}
$$

The real Bott manifold is the product of $S^{1}$ and the 3-dimensional real Bott manifold of Type $(1,1,1)$. 
6 Type $(2,1,1)$

$$
\left(\begin{array}{llll}
0 & 0 & 0 & 1 \\
0 & 0 & 1 & 0 \\
0 & 0 & 0 & 1 \\
0 & 0 & 0 & 0
\end{array}\right)_{2}\left(\begin{array}{llll}
0 & 0 & 1 & 0 \\
0 & 0 & 1 & 1 \\
0 & 0 & 0 & 1 \\
0 & 0 & 0 & 0
\end{array}\right)_{2}\left(\begin{array}{llll}
0 & 0 & 0 & 1 \\
0 & 0 & 1 & 1 \\
0 & 0 & 0 & 1 \\
0 & 0 & 0 & 0
\end{array}\right)_{2}
$$

7 Type $(1,3)$

$$
\left(\begin{array}{llll}
0 & 1 & 1 & 1 \\
0 & 0 & 0 & 0 \\
0 & 0 & 0 & 0 \\
0 & 0 & 0 & 0
\end{array}\right)
$$

8 Type $(1,2,1)$

$$
\left(\begin{array}{llll}
0 & 1 & 1 & 0 \\
0 & 0 & 0 & 0 \\
0 & 0 & 0 & 1 \\
0 & 0 & 0 & 0
\end{array}\right)_{2}\left(\begin{array}{llll}
0 & 1 & 1 & 0 \\
0 & 0 & 0 & 1 \\
0 & 0 & 0 & 1 \\
0 & 0 & 0 & 0
\end{array}\right)_{1}\left(\begin{array}{llll}
0 & 1 & 1 & 1 \\
0 & 0 & 0 & 0 \\
0 & 0 & 0 & 1 \\
0 & 0 & 0 & 0
\end{array}\right)_{2}\left(\begin{array}{llll}
0 & 1 & 1 & 1 \\
0 & 0 & 0 & 1 \\
0 & 0 & 0 & 1 \\
0 & 0 & 0 & 0
\end{array}\right)_{1}
$$

$\mathbf{9}^{\star}$ Type $(1,1,2)$

$$
\left(\begin{array}{llll}
0 & 1 & 1 & 0 \\
0 & 0 & 1 & 1 \\
0 & 0 & 0 & 0 \\
0 & 0 & 0 & 0
\end{array}\right)_{2}
$$

10 Type $(1,1,2)$

$$
\left(\begin{array}{llll}
0 & 1 & 0 & 0 \\
0 & 0 & 1 & 1 \\
0 & 0 & 0 & 0 \\
0 & 0 & 0 & 0
\end{array}\right)_{1}\left(\begin{array}{llll}
0 & 1 & 1 & 1 \\
0 & 0 & 1 & 1 \\
0 & 0 & 0 & 0 \\
0 & 0 & 0 & 0
\end{array}\right)_{1}
$$

11 Type $(1,1,1,1)$

$$
\left(\begin{array}{llll}
0 & 1 & 0 & 1 \\
0 & 0 & 1 & 0 \\
0 & 0 & 0 & 1 \\
0 & 0 & 0 & 0
\end{array}\right)_{1}\left(\begin{array}{llll}
0 & 1 & 1 & 0 \\
0 & 0 & 1 & 1 \\
0 & 0 & 0 & 1 \\
0 & 0 & 0 & 0
\end{array}\right)_{1}\left(\begin{array}{llll}
0 & 1 & 1 & 1 \\
0 & 0 & 1 & 0 \\
0 & 0 & 0 & 1 \\
0 & 0 & 0 & 0
\end{array}\right)_{1}
$$

12 Type $(1,1,1,1)$

$$
\left(\begin{array}{llll}
0 & 1 & 0 & 0 \\
0 & 0 & 1 & 0 \\
0 & 0 & 0 & 1 \\
0 & 0 & 0 & 0
\end{array}\right)_{1}\left(\begin{array}{llll}
0 & 1 & 1 & 0 \\
0 & 0 & 1 & 0 \\
0 & 0 & 0 & 1 \\
0 & 0 & 0 & 0
\end{array}\right)_{1}\left(\begin{array}{llll}
0 & 1 & 0 & 0 \\
0 & 0 & 1 & 1 \\
0 & 0 & 0 & 1 \\
0 & 0 & 0 & 0
\end{array}\right)_{1}\left(\begin{array}{llll}
0 & 1 & 1 & 1 \\
0 & 0 & 1 & 1 \\
0 & 0 & 0 & 1 \\
0 & 0 & 0 & 0
\end{array}\right)_{1}
$$

\section{Riemannian flat real toric manifolds}

A toric manifold $X$ of complex dimension $n$ supports an action of $\left(\mathbb{C}^{*}\right)^{n}$ and its real part $X(\mathbb{R})$ supports an action of $\left(\mathbb{R}^{*}\right)^{n}$, where $\mathbb{C}^{*}=\mathbb{C} \backslash\{0\}$ and $\mathbb{R}^{*}=\mathbb{R} \backslash\{0\}$. Let $T$ be the maximal compact toral subgroup of $\left(\mathbb{C}^{*}\right)^{n}$ and $T^{n}(\mathbb{R})$ be the maximal elementary abelian 2-subgroup of $\left(\mathbb{R}^{*}\right)^{n}$. The orbit space $X(\mathbb{R}) / T^{n}(\mathbb{R})$ can naturally be identified with $X / T$. When $X$ is projective, the orbit space can be identified with a simple $n$-polytope via a moment map. 
The action of $T^{n}(\mathbb{R})$ on the real Bott manifold $M(A)=\mathbb{R}^{n} / \Gamma(A)$ is given as follows. Let $r_{j}(j=1, \ldots, n)$ be the involution on $\mathbb{R}^{n}$ defined by

$$
r_{j}\left(x^{1}, \ldots, x^{n}\right)=\left(x^{1}, \ldots, x^{j-1},-x^{j}, x^{j+1}, \ldots, x^{n}\right) .
$$

Direct computation shows that

$$
r_{j} s_{i}= \begin{cases}s_{i} r_{j} & \text { if } i \neq j, \\ s_{i}^{-1} r_{j} & \text { if } i=j,\end{cases}
$$

where $s_{i}$ is the Euclidean motion on $\mathbb{R}^{n}$ defined in (3-1), so $r_{j}$ induces an involution $\bar{r}_{j}$ on $M(A)=\mathbb{R}^{n} / \Gamma(A)$. Obviously the $\bar{r}_{j}$ commute with each other so that they generate an elementary abelian 2-group of rank $n$ and this gives the action of $T^{n}(\mathbb{R})$.

We remark that the action of $T^{n}(\mathbb{R})$ on $M(A)=\mathbb{R}^{n} / \Gamma(A)$ preserves the flat Riemannian metric on it. The group generated by $s_{i}$ 's and $r_{j}$ 's agrees with the group generated by $r_{j}$ 's and translations by $\frac{1}{2} e^{1}, \ldots, \frac{1}{2} e^{n}$, where $e^{1}, \ldots, e^{n}$ are the standard basis of $\mathbb{R}^{n}$ as before. It follows that the orbit space $M(A) / T^{n}(\mathbb{R})$ can be identified with the $n$-cube

$$
\left\{\left(x^{1}, \ldots, x^{n}\right) \in \mathbb{R}^{n} \mid 0 \leq x^{1} \leq 1 / 2, \ldots, 0 \leq x^{n} \leq 1 / 2\right\} .
$$

The purpose of this section is to prove Theorem 1.2 in the Introduction, that is

Theorem 8.1 A real toric manifold of dimension $n$ which admits a flat Riemannian metric invariant under the action of $T^{n}(\mathbb{R})$ is a real Bott manifold.

We recall some results for the proof of Theorem 8.1. Let $X$ be a toric manifold and let $X_{i}(1 \leq i \leq m)$ be a connected complex codimension-1 closed submanifold of $X$ fixed pointwise under some circle subgroup $T_{i}$ of the torus $T$. We call $X_{i}$ a characteristic submanifold of $X$. Then

$$
K_{X}:=\left\{I \subset\{1, \ldots, m\} \mid \bigcap_{i \in I} X_{i} \neq \varnothing\right\}
$$

is the underlying abstract simplicial complex of the fan of $X$.

Let $X(\mathbb{R})$ be the real part of $X$. The intersection $X_{i} \cap X(\mathbb{R})$ is a connected real codimension-1 closed submanifold of $X(\mathbb{R})$ fixed pointwise under the order 2 subgroup $T_{i}(\mathbb{R}):=T_{i} \cap T^{n}(\mathbb{R})$ of $T^{n}(\mathbb{R})$. Conversely any connected real codimension-1 closed submanifold of $X(\mathbb{R})$ fixed pointwise under an order 2 subgroup of $T^{n}(\mathbb{R})$ is the intersection of $X(\mathbb{R})$ with some $X_{i}$. We call those closed submanifolds characteristic 
submanifolds of $X(\mathbb{R})$ as well. These observations say that there is a bijective correspondence between characteristic submanifolds of $X$ and those of $X(\mathbb{R})$. Hence one can also define $K_{X}$ using the characteristic submanifolds of $X(\mathbb{R})$.

We say that a simplicial complex is a crosscomplex of dimension $n-1$ if it is the boundary complex of a crosspolytope of dimension $n$, where a crosspolytope of dimension $n$ is the dual (or polar) of an $n$-cube. We recall two facts from Masuda and Panov [16]. The first lemma below is stated in [16, Corollary 3.5] in the complex case but it also holds in the real case as stated because of the observation above.

Lemma 8.2 [16, Corollary 3.5] A real toric manifold $X(\mathbb{R})$ is a real Bott manifold if and only if the simplicial complex $K_{X}$ associated with $X(\mathbb{R})$ is a crosscomplex.

Lemma 8.3 [16, Lemma 4.7] Let $K$ be a connected simplicial complex of dimension $k \geq 2$. If the link of each vertex of $K$ is a crosscomplex of dimension $k-1$, then $K$ is a crosscomplex.

Proof of Theorem 8.1 We shall prove the theorem by induction on the dimension $n$. The theorem is obvious when $n=1$. A closed surface which admits a flat Riemannian metric is a torus or a Klein bottle and they are real Bott manifolds, so the theorem also holds when $n=2$.

Now suppose the theorem holds for $n-1 \geq 2$ and let $X(\mathbb{R})$ be a real toric manifold of dimension $n$ which satisfies the assumption in the theorem. Let $X(\mathbb{R})_{1}, \ldots, X(\mathbb{R})_{m}$ be the characteristic submanifolds of $X(\mathbb{R})$. A vertex of the simplicial complex $K_{X}$ associated with $X(\mathbb{R})$ corresponds to some $X(\mathbb{R})_{i}$ and the link of this vertex is the simplicial complex associated with $X(\mathbb{R})_{i}$. Since $X(\mathbb{R})$ admits a Riemannian flat metric invariant under the action of $T^{n}(\mathbb{R})$ and $X(\mathbb{R})_{i}$ is fixed pointwise under the order 2 subgroup $T_{i}(\mathbb{R})$ of $T^{n}(\mathbb{R})$, the metric on $X(\mathbb{R})_{i}$ induced from $X(\mathbb{R})$ is again flat and invariant under the induced action of $T^{n}(\mathbb{R}) / T_{i}(\mathbb{R})$ which is an elementary abelian 2-group of rank $n-1$. Therefore $X(\mathbb{R})_{i}$ is a real Bott manifold by the inductive assumption and hence the link of the vertex of $K_{X}$ is a crosscomplex by Lemma 8.2. Since $\operatorname{dim} K_{X}=n-1 \geq 2, K_{X}$ is a crosscomplex by Lemma 8.3 and hence $X$ is a real Bott manifold by Lemma 8.2. This completes the induction step and the proof of the theorem.

\section{Small covers}

Let $T^{n}(\mathbb{R})$ be an elementary abelian 2-group of rank $n$ as before. A closed smooth manifold $M$ of dimension $n$ with a smooth action of $T^{n}(\mathbb{R})$ is called locally standard 
if each point of $M$ has an invariant open neighborhood equivariantly diffeomorphic to an invariant open subset of a faithful real $T^{n}(\mathbb{R})$-module of dimension $n$. The orbit space of a locally standard $T^{n}(\mathbb{R})$-manifold $M$ is a manifold with corners because the orbit space of a faithful $T^{n}(\mathbb{R})$-module of dimension $n$ is homeomorphic to the product of $n$ half lines. A convex polytope of dimension $n$ is called simple if there are exactly $n$ edges meeting at each vertex, and a simple convex polytope is a typical example of a manifold with corners. If $M$ is locally standard and the orbit space is identified with a simple convex polytope $P$, then $M$ is called a small cover over $P$ (Davis and Januszkiewic [7]). Associated to $P$, there is a closed smooth manifold $\mathbb{R} \mathcal{Z}_{P}$ called the real moment-angle manifold (see [3, Section 6]). It admits an action of $T^{m}(\mathbb{R})$ with $P$ as the orbit space, where $m$ is the number of codimension-1 faces of $P$, and every small cover over $P$ is obtained as the orbit space of $\mathbb{R} \mathcal{Z}_{P}$ by some subgroup of $T^{m}(\mathbb{R})$ of rank $m-n$ acting freely on $\mathbb{R} \mathcal{Z}_{P}$.

A real toric manifold $X(\mathbb{R})$ with the natural $T^{n}(\mathbb{R})$-action is locally standard and its orbit space is often a simple convex polytope. In fact, this is the case when $X$ is projective, so a real toric manifold $X(\mathbb{R})$ is a small cover when $X$ is projective. However there are many small covers which do not arise this way. For example, every closed surface becomes a small cover but only the torus $S^{1} \times S^{1}$ is a real toric manifold among orientable closed surfaces (Payne [21]). We may think of small covers as topological counterparts of real toric manifolds and may ask the cohomological rigidity problem in the Introduction for small covers. We remark that equivariant homeomorphism types of small covers can be distinguished by their equivariant cohomology algebras with $\mathbb{Z} / 2$-coefficients (Masuda [13]).

When $X(\mathbb{R})$ is a real Bott manifold, the orbit space is an $n$-cube as observed in Section 8 ; so a real Bott manifold of dimension $n$ becomes a small cover over an $n$-cube and the converse is known to be true up to homeomorphism.

Theorem 9.1 (Masuda and Panov [16], Choi, Masuda and Suh [5]) A small cover over an $n$-cube is homeomorphic to a real Bott manifold of dimension $n$.

The real moment-angle manifold $\mathbb{R} \mathcal{Z}_{I^{n}}$ associated to an $n$-cube $I^{n}$ is the torus $\mathbb{R}^{n} / \mathbb{Z}^{n}$. The $n$-cube has $2 n$ codimension- 1 faces (so $m=2 n$ ) and the action of $T^{2 n}(\mathbb{R}$ ) on $\mathbb{R} \mathcal{Z}_{I^{n}}=\mathbb{R}^{n} / \mathbb{Z}^{n}$ is induced from the involutions $r_{j}$ 's in (8-1) and the translations by $\frac{1}{2} e_{j}$ 's on $\mathbb{R}^{n}$.

The number $Q_{n}$ of equivariant homeomorphism classes of small covers over an $n$-cube is computed by Choi in [4] for any $n$, eg

$$
Q_{1}=1, Q_{2}=6, Q_{3}=259, Q_{4}=87360, Q_{5}=236240088, \ldots
$$


However, the number $H_{n}$ of (non-equivariant) homeomorphism classes of small covers over an $n$-cube is unknown although

$$
H_{1}=1, H_{2}=2, H_{3}=4, H_{4}=12
$$

as described in Section 7. (Note that the homeomorphism classification is same as the diffeomorphism classification for real Bott manifolds by Theorem 1.1.)

As is well-known, the dodecahedron in dimension 3, the 120-cell in dimension 4, and the $n$-cube and $n$-simplex in any dimension $n$, are regular simple polytopes. The homeomorphism type of a small cover over an $n$-simplex is unique, being that of real projective $n$-space. Small covers over the dodecahedron and the 120-cell admit hyperbolic metrics and are studied in by Garrison and Scott [8] from this point of view. In particular, it is proved that there are exactly 25 small covers over the dodecahedron up to isometry (equivalently up to homeomorphism by Mostow rigidity [18]).

\section{Appendix}

In this appendix we give a proof of the Fact used in Section 6. In fact we will prove a more precise statement. It is well-known that $H_{\phi}^{2}\left(\left(\mathbb{Z}_{2}\right)^{n} ; \mathbb{Z}\right)$ is isomorphic to $(\mathbb{Z} / 2)^{n}$ when $\phi$ is trivial. We prove

Theorem If $\phi$ is non-trivial, $H_{\phi}^{2}\left(\left(\mathbb{Z}_{2}\right)^{n} ; \mathbb{Z}\right)$ is isomorphic to $(\mathbb{Z} / 2)^{n-1}$.

We recall the following Hochschild-Serre spectral sequence, see Mac Lane [12, page 355] or Hochschild and Serre [10].

Proposition Let $1 \rightarrow \Gamma \rightarrow \Pi \rightarrow \Pi / \Gamma \rightarrow 1$ be a group extension and let $A$ be $a$ $\Pi-$ module through a homomorphism $\phi: \Pi \rightarrow \operatorname{Aut}(A)$. Suppose $m \geq 1$ and $H_{\phi}^{q}(\Gamma, A)=0$ for $1<q<m$. For $0<q<m$, there is the exact sequence

$$
\begin{aligned}
0 & \rightarrow H_{\phi}^{1}\left(\Pi / \Gamma, A^{\Gamma}\right) \rightarrow H_{\phi}^{1}(\Pi, A) \rightarrow H_{\phi}^{0}\left(\Pi / \Gamma, H_{\phi}^{1}(\Gamma, A)\right) \rightarrow \\
\cdots & \rightarrow H_{\phi}^{q}\left(\Pi / \Gamma, A^{\Gamma}\right) \rightarrow H_{\phi}^{q}(\Pi, A) \rightarrow H_{\phi}^{q-1}\left(\Pi / \Gamma, H_{\phi}^{1}(\Gamma, A)\right) \\
& \rightarrow H_{\phi}^{q+1}\left(\Pi / \Gamma, A^{\Gamma}\right) \rightarrow H_{\phi}^{q+1}(\Pi, A) \rightarrow \cdots
\end{aligned}
$$

We take $\Pi=\left(\mathbb{Z}_{2}\right)^{n}(n \geq 2)$ and $A=\mathbb{Z}$ as a $\Pi$-module through $\phi: \Pi \rightarrow \operatorname{Aut}(\mathbb{Z})=$ $\{ \pm 1\}$. Choose an order two subgroup $\Gamma \subset\left(\mathbb{Z}_{2}\right)^{n}$ such that $\phi(\Gamma)=\{ \pm 1\}$. Clearly

$$
\Pi=\Gamma \times \operatorname{Ker} \phi .
$$


It is known and easy to check that $H_{\phi}^{2}(\Gamma, A)=0$, so the assumption in the proposition above is satisfied for $m=3$. As $A^{\Gamma}=0$ by our condition, $H_{\phi}^{r}\left(\Pi / \Gamma, A^{\Gamma}\right)=0$ for any $r \geq 0$. Then the exact sequence $\mathrm{A}-1$ becomes

$$
0 \rightarrow H_{\phi}^{2}(\Pi, A) \rightarrow H_{\phi}^{1}\left(\Pi / \Gamma, H_{\phi}^{1}(\Gamma, A)\right) \rightarrow 0 .
$$

On the other hand, it is also known and easy to check that $H_{\phi}^{1}(\Gamma, A) \cong \mathbb{Z} / 2$, so the action of $\Pi / \Gamma$ on $H_{\phi}^{1}(\Gamma, A)$ must be trivial. It follows that

$$
\begin{aligned}
H_{\phi}^{1}\left(\Pi / \Gamma, H_{\phi}^{1}(\Gamma, A)\right) & \cong H^{1}(\Pi / \Gamma, \mathbb{Z} / 2) \\
& \cong H^{1}\left(\left(\mathbb{Z}_{2}\right)^{n-1}, \mathbb{Z} / 2\right) \\
& \cong(\mathbb{Z} / 2)^{n-1} .
\end{aligned}
$$

This together with (A-2) implies the theorem.

Acknowledgments The first author was partially supported by Grant-in-Aid for Scientific Research 2034001 and the second author was partially supported by Grant-in-Aid for Scientific Research 19204007. This work was motivated by a talk of Suyoung Choi on [4] given at Fudan University in January 2008 and very much stimulated by the discussion with Taras Panov which the second author had during his stay at Fudan University. He would like to thank them and also Zhi Lü for inviting him to Fudan University and organizing fruitful seminars. He also would like to thank Dong Youp Suh for comments on an earlier version of this paper and Yasuzo Nishimura for showing him a note concerning matrices discussed in Section 7.

Remark After we completed the first draft of this paper, the second author found the diffeomorphism classification of real Bott manifolds in terms of matrices [14] and a counterexample to the cohomological rigidity problem for real toric manifolds [15].

\section{References}

[1] A Borel, F Hirzebruch, Characteristic classes and homogeneous spaces. I, Amer. J. Math. 80 (1958) 458-538 MR0102800

[2] VM Buchstaber, Circle actions on toric manifolds and their applications, MIMS EPrint: 2006.12 Available at http://eprints.ma.man.ac.uk/108/

[3] V M Buchstaber, T E Panov, Torus actions and their applications in topology and combinatorics, University Lecture Series 24, Amer. Math. Soc. (2002) MR1897064

[4] S Choi, The number of small covers over cubes, Algebr. Geom. Topol. 8 (2008) 23912399 MR2465745 
[5] S Choi, M Masuda, D Y Suh, Quasitoric manifolds over a product of simplices, Osaka J. Math. (to appear) arXiv:0803.2749

[6] S Choi, M Masuda, D Y Suh, Topological classification of generalized Bott towers, Trans. Amer. Math. Soc. (2010)

[7] M W Davis, T Januszkiewicz, Convex polytopes, Coxeter orbifolds and torus actions, Duke Math. J. 62 (1991) 417-451 MR1104531

[8] A Garrison, R Scott, Small covers of the dodecahedron and the 120-cell, Proc. Amer. Math. Soc. 131 (2003) 963-971 MR1937435

[9] M Grossberg, Y Karshon, Bott towers, complete integrability, and the extended character of representations, Duke Math. J. 76 (1994) 23-58 MR1301185

[10] G Hochschild, J-P Serre, Cohomology of group extensions, Trans. Amer. Math. Soc. 74 (1953) 110-134 MR0052438

[11] P Kleinschmidt, A classification of toric varieties with few generators, Aequationes Math. 35 (1988) 254-266 MR954243

[12] S Mac Lane, Homology, Grundlehren series 114, Academic Press, Publishers, New York (1963) MR0156879

[13] M Masuda, Equivariant cohomology distinguishes toric manifolds, Adv. Math. 218 (2008) 2005-2012 MR2431667

[14] M Masuda, Classification of real Bott manifolds arXiv:0809.2178

[15] M Masuda, Cohomological non-rigidity of generalized real Bott manifolds of height 2, Proc. of the Steklov Institute dedicated to the 100th Anniversary of L S Pontryagin (to appear) arXiv:0809.2215

[16] M Masuda, T E Panov, Semi-free circle actions, Bott towers, and quasitoric manifolds, Mat. Sb. 199 (2008) 95-122 MR2452268

[17] M Masuda, D Y Suh, Classification problems of toric manifolds via topology, from: “Toric topology”, Contemp. Math. 460, Amer. Math. Soc. (2008) 273-286 MR2428362

[18] G D Mostow, Quasi-conformal mappings in n-space and the rigidity of hyperbolic space forms, Inst. Hautes Études Sci. Publ. Math. (1968) 53-104 MR0236383

[19] A Nazra, Real Bott tower, Masters thesis, Tokyo Metropolitan University (2008) (A summary can be found in Diffeomorphism type of real Bott towers, Geometry of transformation groups and related topics, RIMS Kôkyûroku No. 1612, Kyoto University, (2008) 165-176)

[20] T Oda, Convex bodies and algebraic geometry, Ergebnisse series 3:15, Springer, Berlin (1988) MR922894 An introduction to the theory of toric varieties, Translated from the Japanese

[21] S Payne, Topology of real toric surfaces arXiv:math.AG/0411265. 
[22] H Sato, Smooth toric Fano five-folds of index two, Proc. Japan Acad. Ser. A Math. Sci. 82 (2006) 106-110 MR2265609

[23] V Uma, On the fundamental group of real toric varieties, Proc. Indian Acad. Sci. Math. Sci. 114 (2004) 15-31 MR2040597

[24] J A Wolf, Spaces of constant curvature, fifth edition, Publish or Perish (1984) MR928600

Department of Mathematics, Tokyo Metropolitan University Minami-Ohsawa 1-1, Hachioji, Tokyo 192-0397, Japan

Department of Mathematics, Osaka City University Sumiyoshi-ku, Osaka 558-8585, Japan

kami@tmu.ac.jp, masuda@sci.osaka-cu.ac.jp

Received: 28 August 2009 